\title{
Avaliação Descritiva sobre o Uso de Esteroides Anabolizantes e seu Efeito sobre as Variáveis Bioquímicas e Neuroendócrinas em Indivíduos que Praticam Exercício Resistido
}

\section{Descriptive Assessment on the Use of Anabolic Steroids and \\ Their Effect on the Biochemical and Neuroendocrine Variables in Practitioners of Resisted Exercise}

Daniel Paulino Venâncio ${ }^{1,3}$

Antonio Claudio Lucas da Nóbrega² Sergio Tufik'

Marco Túlio de Mello1,3

1. Departamento de Psicobiologia

- Unifesp.

2. Universidade Federal Fluminense - UFF.

3. Centro de Estudos em

Psicobiologia e Exercício - CEPE.

Endereço para correspondência: Rua Marselhesa, 535

04020-060 - São Paulo, SP

E-mail: paulinovenancio@gmail.com

\section{RESUMO}

O uso dos esteroides anabolizantes vem se tornando um problema de saúde pública ao longo dos últimos anos. No bojo do uso abusivo, muitos efeitos deletérios são observados, na sua totalidade por disfunções dos vários sistemas fisiológicos. Sendo assim, o objetivo do estudo foi o de avaliar o eixo hipófise-gonadal, a função hormonal, as transaminases hepáticas e o perfil de hemograma de 61 voluntários distribuídos em três grupos: 20 usuários de esteroides anabolizantes praticantes de exercício físico resistido, 21 praticantes de exercício resistido sem uso de esteroides anabolizantes e 20 sedentários. Foi observada elevação do nível de creatina quinase nos dois grupos de indivíduos que se exercitavam de maneira resistida, em relação ao grupo de sedentários $(p<0,001)$. Redução das gonadotrofinas $L H$ e FSH do grupo de usuários de esteroides anabolizantes e elevação do nível de estradiol, em comparação ao grupo sedentário e treinado que não usa esteroides anabolizantes $(p<0,001)$. Ainda, foi observada redução da fração HDL do colesterol, em relação aos dois grupos estudados $(p<0,001)$. Desta maneira, o uso dos esteroides anabolizantes causa alterações bioquímicas que podem levar a instalação de efeitos colaterais.

Palavras-chave: esteróides anabolizantes, exercício físico resistido, efeitos adversos.

\section{ABSTRACT}

The use of anabolic asteroids has become a public health problem over the last years. Concerning their abusive use, many deleterious effects caused in their totality by dysfunction of the various physiological systems can be observed. Therefore, the aim of the present study was to assess the hypophyseal-gonadal axis, hormone function, hepatic transaminases and hemogram profile of 61 volunteers distributed in three groups: 20 anabolic steroid users, practitioners of resisted physical exercise; 21 practitioners of resisted physical exercise with no use of anabolic steroids and 20 sedentary subjects. Increase of the creatine kinase level was observed in the two exercised groups in comparison to the sedentary group $(p<0.001)$. Reduction of the $\mathrm{LH}$ and FSH gonadotrpins of the steroid users group and increase in the estradiol level were observed in comparison to the sedentary and with no steroid use groups $(p<0.001)$. Moreover, reduction of the HDL cholesterol fraction was observed in comparison to the two studied groups ( $p<$ 0.001). Thus, the use of anabolic steroids causes biochemical alterations which can lead to the installation of collateral effects.

Keywords: anabolic androgenic sterois, resistance exercise, side effects. 


\section{INTRODUÇÃO}

Os esteroides anabolizantes são drogas sinteticamente derivadas da testosterona, o hormônio sexual masculino ${ }^{(1,2)}$. Nas últimas décadas essas drogas vêm sendo utilizadas por atletas de elite, em maior parte, aqueles envolvidos em esportes de força e velocidade, para melhora do desempenho físico nas competições ${ }^{(2-4)}$. Entretanto, o abuso dos esteroides anabolizantes passou a ser feito por frequentadores de academias, mais interessados nas alterações provocadas na composição corporal, observados com o aumento da massa magra e na redução da gordura subcutânea(2,4-6).

Atualmente, nos EUA, estima-se que haja 3,5 milhões de usuários de esteroides anabolizantes e que, aproximadamente 3\% dos jovens norte-americanos já fizeram uso dessa classe de drogas em algum período da vida ${ }^{(5)}$. No Brasil, o levantamento anual sobre o uso de drogas psicotrópicas pelos jovens brasileiros em idade escolar, nas principais capitais brasileiras, demonstrou que, aproximadamente, 2\% deles já haviam feito uso dos esteroides anabolizantes.

Na última década, houve um avanço sobre os mecanismos fisiológicos e moleculares responsáveis pela ação dos esteroides anabolizantes sobre o sistema musculoesquelético, influenciando a massa magra, o tamanho muscular, a força, o metabolismo de proteína e o metabolismo ósseo ${ }^{(1,2)}$. Sendo assim, o tecido muscular é o tecido alvo para ação anabólica dessa classe de substância. Os efeitos anabólicos da testosterona foram comprovados em um estudo elegante de Bhasin et al. (1996), demonstrando que voluntários normais, quando recebiam testosterona e faziam treinamento de força concomitantemente, aumentavam a força e a secção transversa do músculo. Mais recentemente, foi observada relação de dose-resposta entre a força e a área transversa do músculo de jovens normais que recebiam testosterona ${ }^{(7,8)}$. Postula-se que existam dois mecanismos responsáveis pela indução da hipertrofia muscular pelo uso de esteroides anabolizantes: o mecanismo direto e o indireto ${ }^{(9)}$.

O mecanismo direto é mediado pela interação do hormônio com o receptor androgênico, localizado no citoplasma. Após essa interação e subsequente translocação para regiões específicas do núcleo, ocorre a sinalização para produção de proteínas ${ }^{(9)}$. 0 treinamento e o uso de esteroides anabolizantes aumentam o número de receptores androgênicos no tecido muscular, demonstrando a importância fisiológica dessa proteína para a hipertrofia(9-11).

O mecanismo indireto não depende da ativação do receptor androgênico pelo hormônio, mas sim, pela interação com outros fatores tróficos, como o IGF-I e, também, por meio da interação dos esteroides anabolizantes com o receptor de glicocorticoide, inibindo a degradação de proteínas ${ }^{(1,12)}$.

Porém, o uso dos esteroides anabolizantes, indiscriminadamente, causa uma série de efeitos colaterais. Recentemente, foi constatado que quase 100\% dos usuários de esteroides anabolizantes apresentavam algum efeito colateral. Dentre eles, os mais comuns são acne, atrofia testicular, retenção hídrica, alterações do humor e ginecomastia ${ }^{(1,13)}$. Além disso, existe grande alteração das variáveis bioquímicas com o uso dos esteroides anabolizantes, como hormônios do eixo hipotálamo-hipófise-gonadal, enzimas hepáticas, células do sistema hematopoietico e perfil lípidico sanguíneo, amiúde referido como fator de risco para o aparecimento de doenças cardiovasculares.

A proposta do estudo foi a de avaliar, de forma transversal, o perfil hormonal e bioquímico de usuários de esteroides anabolizantes com seus hábitos de uso e regime de treinamento.

\section{MÉTODOS}

\section{Voluntários}

Após a aprovação de todos os procedimentos experimentais do estudo pelo Comitê de Ética em Pesquisa da Universidade Federal de São Paulo (no 1.309/02), três grupos de indivíduos do gênero masculino foram recrutados para participar do estudo: a) 20 indivíduos, de $26 \pm$ 1,2 anos de idade, todos utilizando esteroides anabolizantes por via oral ou injetável, praticantes de exercício físico resistido; b) 21 indivíduos, de $26,01 \pm 1,0$ ano de idade, que nunca utilizaram esteroides anabolizantes, mas praticavam exercício físico resistido; e c) 20 indivíduos sedentários que nunca utilizaram esteroides anabolizantes.

\section{Processo de recrutamento}

A seleção dos voluntários foi realizada por meio de anúncios, nas academias de ginástica da cidade de São Paulo, e também em fóruns de discussão sobre uso de esteroides anabolizantes na internet. Os critérios de exclusão adotados foram: usuários de drogas psicotrópicas, indivíduos que consumiam mais de 15 doses de bebida alcoólica por semana ( $\cong 30 \mathrm{~g} / \mathrm{dia}$ ). Ainda, foram prontamente excluídos do estudo trabalhadores em turno, indivíduos que haviam realizado viagens transmeridionais nos últimos 12 meses e portadores de doenças metabólicas.

Os usuários de esteroides anabolizantes adquiriam as drogas de fontes não-médicas, a duração média do ciclo de uso foi de $9 \pm 2,56$ semanas. Os indivíduos autoadministravam as drogas, a dosagem semanal média foi de 653,4 \pm 99,47mg de vários compostos diferentes (tabela 1). Os voluntários administravam dois ou mais tipos de esteroides anabolizantes simultaneamente com variação na dosagem utilizada ao longo das semanas.

Os voluntários selecionados para o grupo de usuários tinham total responsabilidade pelo uso da substância e nunca foram encorajados a fazê-lo. Além disso, foram esclarecidos dos possíveis efeitos deletérios da administração dessas drogas ao organismo, estando cientes dos riscos.

\section{Análise bioquímica}

Os voluntários foram submetidos à punção da veia anticubital do braço direito para retirada de amostras de sangue para análise do eixo hipotálamo-hipófise-gonadal, enzimas hepáticas, perfil lipídico, hormônio tireoidiano, fator de crescimento derivado da insulina I (IGF-I), glicemia e células sanguíneas.

Todo o sangue foi processado e centrifugado a $3.000 \mathrm{rpm}$ por $15 \mathrm{~min}$, o soro e o plasma foram pipetados e aliquotados em tubos de ensaios e estocados a $-80^{\circ} \mathrm{C}$ até análise posterior. A testosterona total ( $\mathrm{T}$ ), o estradiol (E2), o hormônio luteinizante (LH), o hormônio folículo estimulante (FSH) foi mensurado pelo método de quimioluminescência (Advia Centaur, Bayer Corporation, Tarrytown, NY, EUA). O limiar de detecção foi de 10ng/dl para a T (Ortho-Climinal Diagnostics, Inc Amersham, Inglaterra), 7,0pg/dl para a E2 (Advia Centaur, Bayer Corporation, Tarrytown, NY, EUA), $0,3 \mathrm{mU} / \mathrm{mL}$ para o FSH e 0,07mU/mL para o LH. Ainda, foi utilizado para a dosagem do cortisol e do hormônio adrenocorticotrófico (ACTH) o método de radioimunoensaio.

Foram avaliadas as enzimas hepáticas TGO, TGP, aldolase e creatina quinase (CK) para quantificar os efeitos hepáticos do uso dos esteroides anabolizantes, além das plaquetas, eritrócitos e hemoglobina, proteínas do sistema hematopoiético altamente responsivas ao uso dos esteroides anabolizantes.

\section{Análise estatística}

Os dados estão representados como média \pm erro padrão. Foi utilizado o teste de Anova de uma via, seguido do teste de Tukey para comparação entre os grupos. O nível de significância foi determinando em $(p<0,05)$. 


\section{RESULTADOS}

\section{Eixo hipotálamo-hipófise-gonadal}

O teste de Anova para a concentração de T, E2, LH e FSH revelou significante alteração da concentração hormonal desse eixo $\left(F_{5,31}=\right.$ 0,46; $p<0,0001)$. O teste de post-hoc revelou uma drástica redução da concentração de LH e FSH no grupo de usuários de esteroides anabolizantes quando comparado aos grupos de indivíduos sedentários e treinados. Não houve diferença estatisticamente significativa entre os grupos sedentários e treinados (figura 1).

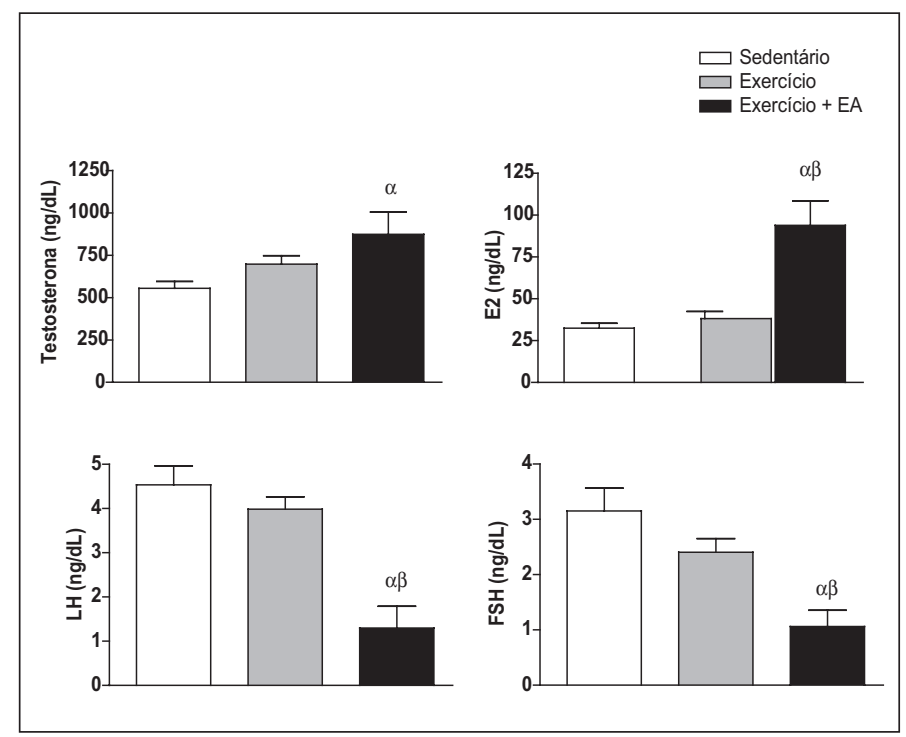

Figura 1. As barras dos gráficos representam a média \pm erro padrão da média para a concentração plasmática de testosterona total, estradiol (E2) hormônio luteinizante (LH) e hormônio folículo estimulante (FSH). $\alpha$ diferente do grupo sedentário e $\beta$ diferente do grupo exercício.

\section{ACTH e cortisol}

Não foi detectado nenhum efeito do uso dos esteroides anabolizantes e do treinamento resistido sobre as concentrações plasmáticas de cortisol e ACTH dos três grupos estudados (figura 2).

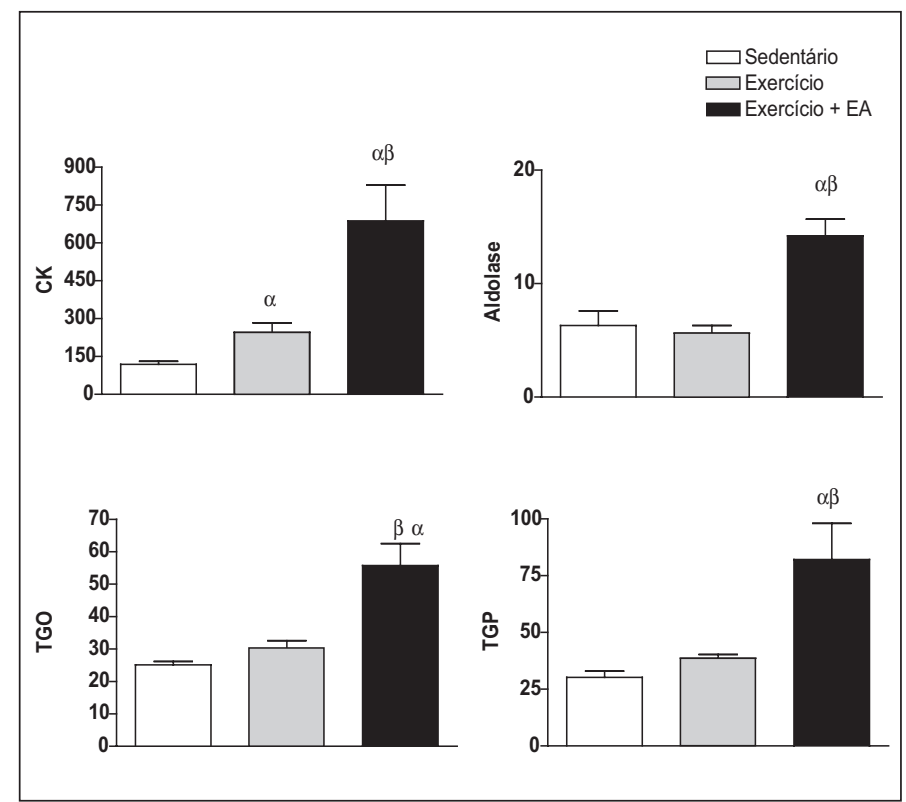

Figura 2. Avaliação das enzimas hepáticas. Creatina quinase (CK), aldolase, transamina glutamil oxidase (TGO) e transamina glutamil peroxidase (TGP). $\alpha$ diferente do grupo sedentário e $\beta$ diferente do grupo controle.

\section{Enzimas hepáticas}

O grupo de usuários de esteroides anabolizantes apresentou alteração na concentração das enzimas hepáticas $\left(F_{58,72}=0,16 ; p<0,001\right)$. A avaliação desse efeito revelou significativa diferença entre o grupo exercitado que utilizou esteroides anabolizantes comparado ao grupo sedentário e exercício nas concentrações das enzimas TGO, TGP e CK e aldolase $(p<0,0001)$ (figura 3).

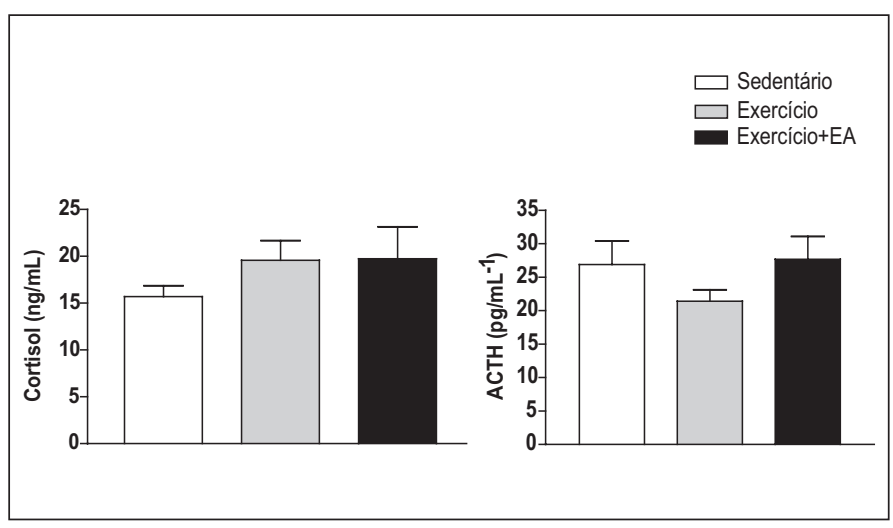

Figura 3. As barras dos gráficos representam a média \pm erro padrão da média para a concentração plasmática de cortisol e do ACTH.

\section{TSH, T4 livre e IGF-I}

Não foi observada nenhuma diferença sobre a concentração de TSH, e T4 livre entre os grupos estudados. Já com relação à concentração plasmática de IGF-I, o teste de Anova detectou diferença significativa entre os grupos estudados $\left(F_{2149}=97,4 ; p<0,001\right)$. A análise de post-hoc revelou diferença significativa entre os grupos exercitado e exercitado que utilizou esteroides anabolizantes em relação ao grupo que não utilizou ( $<<0,0001$ ) (figura 4).

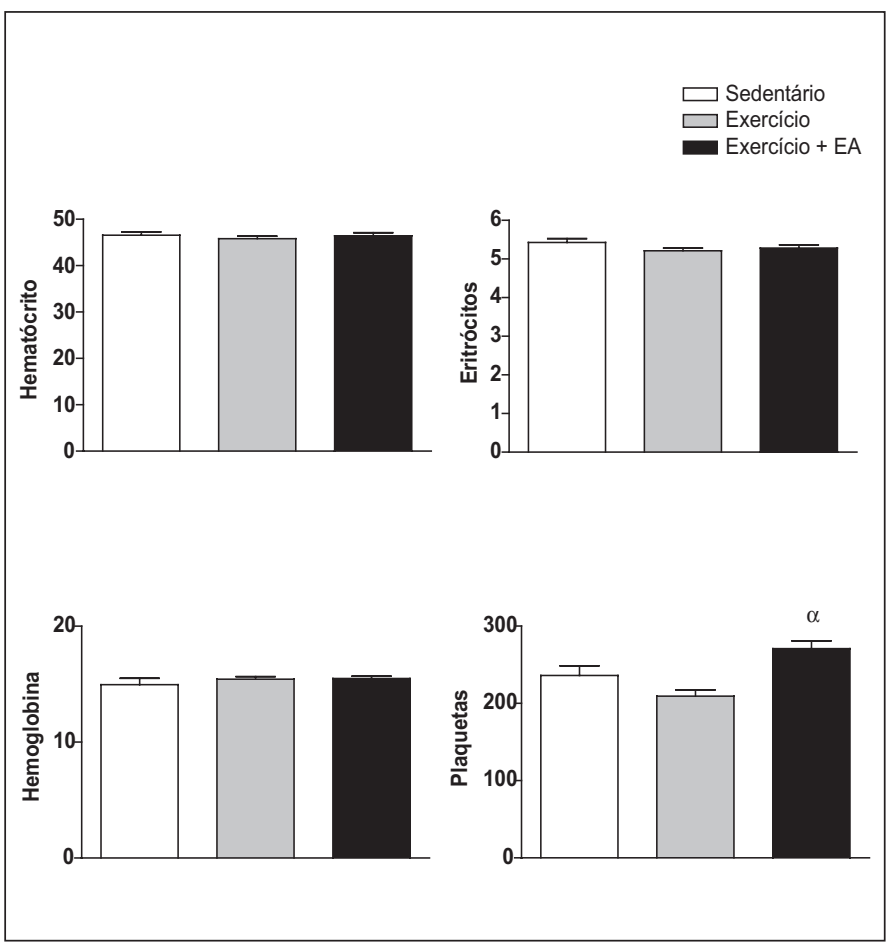

Figura 4. As barras dos gráficos representam a média \pm erro padrão da média para a concentração plasmática de hmatócrito, eritrócito, hemoglobina e plaquetas. $\alpha$ diferente do grupo de sedentários. 


\section{Hematócrito, hemoglobina, eritrócitos e plaquetas}

Um significante efeito foi observado nos níveis de plaquetas $\left(F_{2,51}=8,97 ; p<0,001\right)$. A análise de post-hoc revelou diferença significativa do grupo que fez exercício e utilizou esteroides anabolizantes em relação ao grupo que apenas fez exercício $(p<0,0001)$. Ainda, foi observada tendência à diferença significativa na análise de post-hoc entre o grupo que fez uso da droga e o grupo sedentário $(p=0,054)$ (figura 5).

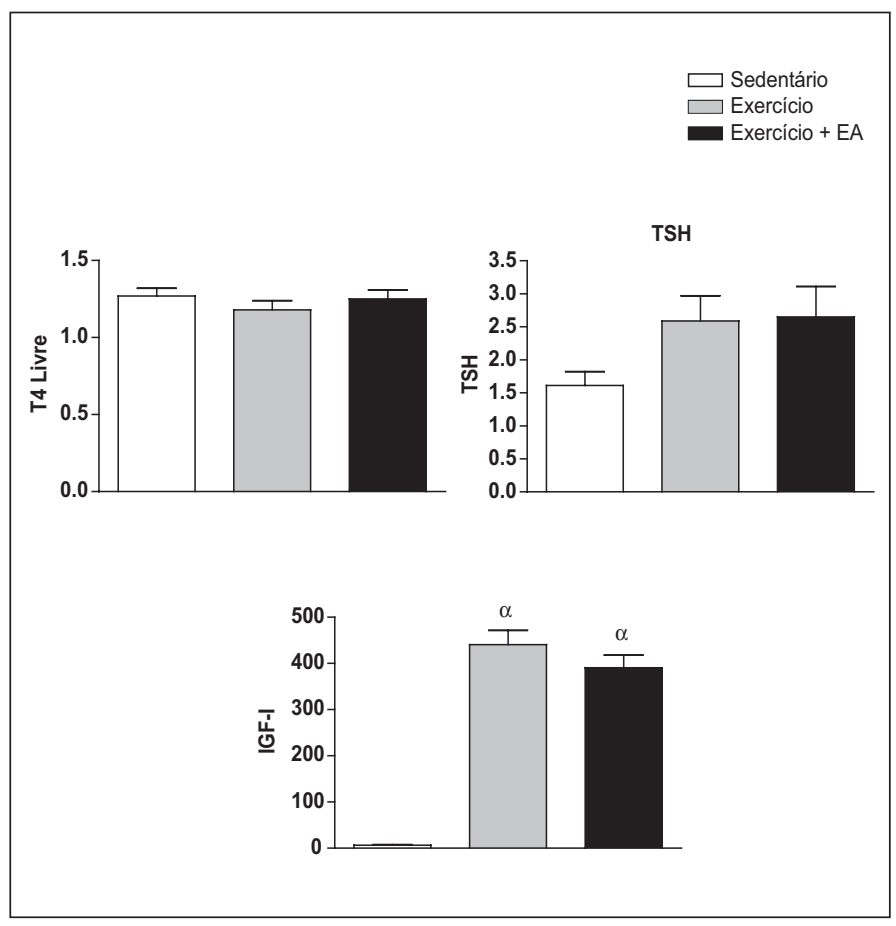

Figura 5. As barras dos gráficos representam a média \pm Erro padrão da média para a concentração plasmática de T4 livre, TSH e IGF-I. $\alpha$ diferente do grupo sedentário.

\section{Glicemia, HDL, LDL, colesterol, triglicérides, ureia e creatinina}

Foi observada diferença estatisticamente significativa entre as variáveis estudadas $\left(F_{3,32}=0,43 ; p<0,001\right)$. A análise desse efeito revelou significativa redução na concentração de HDL entre o grupo de usuários em relação aos grupos sedentário e exercício sem esteroides anabolizantes $(p<0,0001)$. Ainda, tanto o grupo exercício quanto grupo que utilizou esteroides anabolizantes apresentou maiores concentrações de LDL em relação ao grupo sedentário $(p<0,001)$ e o grupo exercício apresentou maiores níveis de colesterol plasmático em comparação ao grupo sedentário $(p<0,001)$ (tabela 1$)$.

Tabela 1. Variáveis bioquímicas

\begin{tabular}{c|c|c|c|c}
\hline Sedentário & Sedentário & Exercício & Exercício + EA & $\mathbf{p}$ \\
\hline Glicemia & $84,82 \pm 2,01$ & $84,65 \pm 1,48$ & $80,25 \pm 2,12$ & NS \\
\hline Ureia & $27,82 \pm 1,20$ & $33,16 \pm 1,52$ & $32,78 \pm 2,49$ & NS \\
\hline Creatinina & $1,03 \pm 0,03$ & $1,03 \pm 0,03$ & $1,09 \pm 0,04$ & NS \\
\hline HDL & $53,47 \pm 2,73$ & $59,65 \pm 3,76$ & $34,05 \pm 3,11 \alpha \beta$ & $<0,0001$ \\
\hline LDL & $72,82 \pm 4,42$ & $94,65 \pm 4,63 \alpha$ & $97,71 \pm 6,31 \alpha$ & $<0,001$ \\
\hline Triglicérides & $71,88 \pm 7,26$ & $77,35 \pm 5,06$ & $91,90 \pm 10,21$ & NS \\
\hline Colesterol & $140,65 \pm 5,21$ & $169,82 \pm 6,95 \alpha$ & $150,05 \pm 6,91$ & $<0,001$ \\
\hline
\end{tabular}

Os valores são expressos como média \pm erro padrão da média. $\alpha$ diferente do grupo de sedentários; $\beta$ diferente do grupo exercício.

\section{DISCUSSÃO}

Estudos demonstrando os efeitos do uso de doses suprafarmacológicas de esteroides anabolizantes são de grande importância para elucidação dos principais parâmetros fisiológicos acometidos com o uso abusivo. Nesse sentido, nosso estudo demonstra a clara relação existente entre o uso abusivo dos esteroides anabolizantes e o comprometimento de algumas variáveis bioquímicas, hematológicas e hormonais.

Desta maneira, o grupo de usuários de esteroides anabolizantes apresentou destacada redução na fração HDL do colesterol e das gonadotrofinas LH e FSH. Ainda, foi observada elevação no nível plasmático de CK, TGO e TGP, estradiol, e IGF-I, este último, nos dois grupos que faziam exercício resistido.

O uso dos esteroides anabolizantes levou a supressão da liberação das gonadotrofinas LH e FSH, que é um efeito reportado em muitos estudos na literatura ${ }^{(14-16)}$. Particularmente, a redução dos níveis de FSH pode levar a diminuição da contagem, da mobilidade e alteração da morfologia dos espermatozoides ${ }^{(17)}$. Em relação ao LH, essa redução leva a alteração da sinalização para a síntese e posterior liberação de testosterona; possivelmente, essa é uma explicação para os baixos níveis de testosterona encontrados em usuários de esteroides anabolizantes, dependendo da classe de sustâncias utilizadas.

Adicionalmente a isso, os indivíduos do grupo que faziam uso dos esteroides anabolizantes apresentaram elevado nível de E2. Os elevados níveis de testosterona são biotransformados pela enzima aromatase, elevando o nível de estradiol. As repercussões dessa alteração dos níveis de 22 podem ir desde ao fechamento prematuro das epífises ósseas, quando indivíduos que ainda não completaram o crescimento, a alterações comportamentais, como instalação de ansiedade e depressão(1). Além disso, é bastante comum, por conta da elevação dos níveis de estradiol, os usuários apresentarem acúmulo de gordura em determinados sítios do corpo, como por exemplo, na região dos mamilos. Isso leva a instalação da ginecomastia que, dependendo da extensão, são minimizadas ou retiradas apenas com intervenção cirúrgica.

A literatura demonstra a relação entre o uso de esteroides anabolizantes e o aumento da atividade de enzimas hepáticas, particularmente com os esteroides da classe dos 17 -a-alquilados ${ }^{(18)}$. As drogas injetáveis, como a testosterona cipionato e as preparações em enantato, parecem não afetar a função hepática. No entanto, são reportados casos de adenoma hepático com o uso dessas substâncias ${ }^{(19)}$. Urhausen et al. estudaram a reversibilidade dos efeitos do uso dos esteroides anabolizantes em inúmeras variáveis, entre elas, algumas enzimas associadas à função hepática. Nesse sentido, ficou evidenciado que, com o uso de esteroides, houve elevação dos níveis de CK, CK fração miocárdica, GGT ${ }^{(20)}$. Em nosso estudo, também observamos elevação dos níveis de CK, em ambos os grupos, treinados e treinados com o uso dos esteroides anabolizantes. O efeito da intensidade de treinamento para essa variável em específico parece ser mais fortemente associada ao fator intensidade de treino do que ao uso dos esteroides anabolizantes, sobretudo porque o grupo que fez uso da substância, na literatura, parece, comprovadamente, treinar de maneira mais intensa se comparado aos indivíduos que apenas treinam e não utilizam essas drogas ${ }^{(1)}$. O mesmo parece ocorrer para as outras transaminases avaliadas em nosso protocolo, visto que o exercício físico resistido pode, em grande maneira, elevar o nível dessas enzimas ${ }^{(21)}$. Entretanto, o treinamento resistido per se pode elevar os níveis de algumas transaminases, como a $\mathrm{CK}^{(21,22)}$. 
Os dados de vários autores sugerem que as alterações no perfil lípidico com uso dos esteroides anabolizantes ocorrem em grande parte na fração HDL do colesterol, principalmente, com o uso dos esteroides anabolizantes da classe dos 17-a-alquilados ${ }^{(23-26)}$.

Podemos considerar que o uso dos esteroides anabolizantes causa efeitos deletérios sobre inúmeras variáveis fisiológicas. Além disso, o uso abusivo dessas substâncias, em específico, pode levar ao comprometimento da função neuroendócrina, com o aparecimento de efeitos colaterais decorrentes da mudança desse balanço hormonal e prejudicar a capacidade reprodutiva do homem, potencializar o aparecimento de câncer na próstata e ginecomastia. Não obstante, parece que esses efeitos podem ser minimizados com a parada total do uso. No entanto, estudos Iongitudinais são necessários para elucidar o período exato do aparecimento dessas alterações, sobretudo com controle de classes de drogas utilizadas pelos voluntários.

\section{AGRADECIMENTOS}

Os autores são gratos a AFIP, CEPE, CENSA, CEPID/Fapesp-Sono, Fada-Unifesp e CNPq pelo apoio financeiro.

Todos os autores declararam não haver qualquer potencial conflito de interesses referente a este artigo.

\section{REFERÊNCIAS}

1. Hartgens F, Kuipers H. Effects of androgenic-anabolic steroids in athletes. Sports Med 2004;34:513-54.

2. Wilson JD. Androgen abuse by athletes. Endocr Rev 1988;9:181-99.

3. Yesalis CE, Bahrke MS. Anabolic-androgenic steroids and related substances. Curr Sports Med Rep 2002;1:246-52.

4. Yesalis CE III, Wright JE, Bahrke MS. Epidemiological and policy issues in the measurement of the long term health effects of anabolic-androgenic steroids. Sports Med 1989;8;129-38.

5. Eklof AC, Thurelius AM, Garle M, Rane A, Sjoqvist F. The anti-doping hot-line, a means to capture the abuse of doping agents in the Swedish society and a new service function in clinical pharmacology. Eur J Clin Pharmacol 2003;59:571-7.

6. Pope HG Jr, Katz DL. Psychiatric and medical effects of anabolic-androgenic steroid use. A controlled study of 160 athletes. Arch Gen Psychiatry 1994;51:375-82.

7. Bhasin S, Storer TW, Berman N, Callegari C, Clevenger B, Phillips J, et al. The effects of supraphysiologic doses of testosterone on muscle size and strength in normal men. N Engl J Med 1996;335:1-7.

8. Bhasin S, Woodhouse L, Casaburi R, Singh AB, Bhasin D, Berman N, et al. Testosterone dose-response relationships in healthy young men. Am J Physiol Endocrinol Metab 2001;281:E1172-81.

9. Sheffield-Moore M, Urban RJ. An overview of the endocrinology of skeletal muscle. Trends Endocrinol Metab 2004;15:110-5.

10. Kadi F. Adaptation of human skeletal muscle to training and anabolic steroids. Acta Physiol Scand Suppl 2000;646,1-52

11. Kadi F, Bonnerud P, Eriksson A, Thornell LE. The expression of androgen receptors in human neck and limb muscles: effects of training and self-administration of androgenic-anabolic steroids. Histochem Cell Biol 2000;113:25-9.

12. Sheffield-Moore M. Androgens and the control of skeletal muscle protein synthesis. Ann Med. 2000;32:181-6

13. Parkinson AB, Evans NA. Anabolic androgenic steroids: a survey of 500 users. Med Sci Sports Exerc. 2006;38:644-51
14. Alen M, Reinila M, Vihko, R. Response of serum hormones to androgen administration in power athletes. Med Sci Sports Exerc 1985;17:354-9.

15. Clerico A, Ferdeghini M, Palombo C, Leoncini R, Del Chicca MG, Sardano G, et al. Effect of anabolic treatment on the serum levels of gonadotropins, testosterone, prolactin, thyroid hormones and myoglobin of male athletes under physical training. J Nucl Med Allied Sci 1981;25:79-88.

16. Small M, Beastall GH, Semple CG, Cowan RA, Forbes CD. Alteration of hormone levels in normal males given the anabolic steroid stanozolol. Clin Endocrinol (Oxf) 1984;21:49-55.

17. Bonetti A, Tirelli F, Catapano A, Dazzi D, Dei CA, Solito F, et al. Side effects of anabolic androgenic steroids abuse. Int J Sports Med 2008;29:679-87.

18. Marquardt GH, Logan CE, Tomhave WG, Dowben RM. Failure of non-17-alkylated anabolic steroids to produce abnormal liver function tests. J Clin Endocrinol Metab 1964;24:1334-6.

19. Shapiro P, Ikeda RM, Ruebner BH, Connors MH, Halsted CC, Abildgaard CF. Multiple hepatic tumors and peliosis hepatis in Fanconi's anemia treated with androgens. Am J Dis Child 1977;131:1104-6.

20. Urhausen A, Torsten A, Wilfried K. Reversibility of the effects on blood cells, lipids, liver function and hormones in former anabolic-androgenic steroid abusers. J Steroid Biochem Mol Biol 2003;84:369-75.

21. Noakes TD. Effect of exercise on serum enzyme activities in humans. Sports Med 1987;4:245-67.

22. Dickerman RD, Pertusi RM, Zachariah NY, Dufour DR, McConathy WJ. Anabolic steroid-induced hepatotoxicity: is it overstated? Clin J Sport Med 1999;9:34-9.

23. Bronson FH. Effects of prolonged exposure to anabolic steroids on the behavior of male and female mice. Pharmacol Biochem Behav 1996;53:329-34.

24. Bronson FH, Matherne CM. Exposure to anabolic-androgenic steroids shortens life span of male mice. Med Sci Sports Exerc 1997;29:615-9.

25. Taggart HM, Applebaum-Bowden D, Haffner S, Warnick GR, Cheung MC, Albers JJ, et al. Reduction in high density lipoproteins by anabolic steroid (stanozolol) therapy for postmenopausal osteoporosis. Metabolism 1982;31:1147-52.

26. Thompson PD, Cullinane EM, Sady SP, Chenevert C, Saritelli AL, Sady M, et al. Contrasting effects of testosterone and stanozolol on serum lipoprotein levels. JAMA 1989;261:1165-8. 